\title{
Le Système d'Innovation National appliqué à l'agriculture : une proposition méthodologique de diagnostic en Afrique
}

\author{
The National Innovation System as applied to Agriculture: \\ a methodological proposal for diagnosis in Africa
}

\author{
Sawadogo/Compaore MFW Eveline ${ }^{1}$, Sawadogo Natewinde ${ }^{2}$ \\ ${ }^{1}$ Institut de l'Environnement et de Recherches Agricoles, Burkina Faso, compeve@yahoo.fr \\ ${ }^{2}$ Université Ouaga II, Burkina Faso, natewinde.sawadogo@yahoo.fr
}

\begin{abstract}
RÉSUMÉ. Les interventions politiques dans le secteur agricole n'ont pas toujours été instruites par des méthodologies appropriées afin de réduire les risques d'échec. En Afrique, les interventions dans le secteur de l'agriculture reposent depuis longtemps sur deux approches principales. II y a d'abord l'approche linéaire (top-down) de développement, puis l'approche participative (top-down-bottom-up) ou systémique. Cependant, les modèles linéaires de diffusion de l'innovation sont devenus obsolètes. Aussi, au sein des modèles interactionnels, certains se distinguent des autres. C'est le cas du cadre du Système d'Innovation National (SIN) qui est un outil d'analyse et de gouvernance politique dans de nombreux pays africains, y compris le Burkina Faso. Dans ce dernier cas, le cadre (SIN) est testé depuis le début des années 2000. De nos jours, il est très populaire dans le milieu politique et de recherche. L'agriculture étant un secteur clé des pays africains comme le Burkina Faso, le défi actuel consiste à élaborer un guide méthodologique pratique pour le diagnostic des systèmes d'innovation agricoles en Afrique. Cela nous amène à une contextualisation des outils fournis par le modèle. Ce présent article est une contribution qui s'inspire des résultats de recherches des auteurs. La première partie de l'article présente la démarche mobilisée. Ensuite, il fait l'état des approches sur l'innovation agricole au Burkina Faso et en Afrique en général et leurs fondements théoriques. Enfin, il fait une proposition méthodologique de diagnostic des systèmes d'innovation agricoles au Burkina Faso et en Afrique.

ABSTRACT. Policy interventions in the agricultural sector have not always been informed by methodologies to reduce the risk of failure. In Africa, interventions in the agricultural sector have long depended on two main approaches. First there is the linear approach (top-down) of development, and then the participative (top-down-bottom-up) or systemic approach. However, linear models of diffusion of innovation have become obsolete. Also, within the interactive models, there are some differences. It is the National System of Innovation (NIS) system that is established as an analytical and policy tool in many African countries, including Burkina Faso. The NIS has been tested since the early 2000 s and is currently very popular in policy setting and research contexts. Agriculture being a key sector for African countries, the current challenge is to develop a practical methodological guide for the diagnosis of agricultural innovation systems in Africa. This paper is intended to be a contribution that draws on the research results of the authors to address this methodological challenge. The first part of the paper presents the approach used. Then, it sheds light on the agricultural innovation approaches in Burkina Faso and in Africa in general, and their theoretical foundations. Finally, it proposes a methodological approach for diagnosis of the agricultural innovation systems in Africa.

MOTS-CLÉS. Méthodologie, Système d'innovation agricole, Burkina Faso.

KEYWORDS. Methodology, Agricultural innovation system, Burkina Faso.
\end{abstract}

\section{Introduction}

Depuis la fin des années 1990, «le modèle du marché » commence à perdre sa legitimité en tant que cadre de régulation dominant du développement (Compaore 2015; Sawadogo et Sawadogo/Compaore 2018). En effet le rapport de la Banque Mondiale sur le développement de 1998 s'intitulait Knowledge for Development (World Bank, 1998); l'année suivante, celui de l'UNESCO avait pour titre Science in the Twenty-first Century, A New Commitment. Pour tout observateur averti des questions de développement, il est clair que le Consensus de Washington qui 
consacra le modèle du marché était en crise, et qu'un nouveau consensus se mettait en place (Cimoli et al. 2009). Ainsi, le nouveau millenaire débute en Afrique par le déploiement d'un nouveau modèle de développement qui accorde desormais une place importante à l'innovation. Il s'agit d'un modèle de développement basé sur la connaissance (knowledge-based development) qui, dès 1998, donne lieu à plusieurs rencontres sur le continent Africain. Dès lors, en 1999, pendant l'année de publication du rapport de l'UNESCO ci-dessus mentionné, le réseau Knowledge Management in Africa (KMA) organisa un colloque en Afrique du Sud sur le thème 'Knowledge to Address Africa's Developpement Challenges'. Deux années plus tard, la création du Nouveau Partenariat pour le Développement de l'Afrique (NEPAD) se fixe pour objectif de mettre en place de nouvelles conditions pour le développement en Afrique. En 2003, le Conseil Ministériel Africain sur la Science et la Technologie au cours de sa première rencontre à Johannesburg en Afrique du Sud a réaffirmé le rôle de la science dans le développement du continent africain. En Janvier 2007, ce processus a été couronné par la déclaration d'Addis-Abeba sur la Science, la Technologie, et la recherche scientifique pour le développement lors du Sommet de l'Union Africaine. Dès lors, dans tous les secteurs, et celui de l'agriculture en particulier, de nouveaux concepts commencèrent à pénétrer les discours et les pratiques, notamment à travers l'usage du cadre d'analyse du Système d'Innovation National (SIN). Ce cadre, élaboré par Freeman et Lundvall (1988), est un modèle concomitant d'analyse et d'intervention qui fait de l'innovation le moteur du développement économique. C'est depuis le début des années 2000 que le SIN a été testé. Il est devenu très populaire dans les contextes des politiques publiques et de ceux de la recherche en Afrique (Muchie et all 2003 ; Touzard et all, 2015).

Cependant la diffusion de ce nouveau modèle de développement dans l'agriculture reste un défi pour les acteurs (chercheurs, praticiens du dévéloppement et politiques) du secteur. En effet, la conception linéaire et diffusioniste de l'innovation a profondemment marqué les structures d'enseignements, la gouvernance publique et les référentiels cognitifs sans résultats positifs notables au regard des indicateurs de développement actuels. La recherche d'une alternative restait encore au début du $21^{\text {ème }}$ siècle plus à l'état «d'expression d'intérêt » que de propositions concrètes de méthodes et d'outils pratiques permettant d'analyser un Système d'Innovation Agricole (SIA) (OECD, 2013) et de sa transformation pour le développement. Les propos d'un agronome Francais à la fin des années 1990 en sont assez illustratifs:

les Interventions des pouvoirs publics [...] resteront sans doute encore nécessaires pendant longtemps. Mais il apparaît desormais de refondre totalement la démarche et les méthodes relatives à la conception, la formulation, la mise en œuvre, le suivi et l'evaluation des projets en tirants les leçons des expériences passées. Il importe surtout de concevoir des procedures democratiques qui permettront de réellement prendre en compte les intérêts respectifs des diverses classes, couches et cathégories sociales [...] (Dufumier 1996:10)

Ces propos font alors appel à la nécessité de passage d'une approche de développement descendante à une approche de développement participative et démocratique. Ils réferrent à l'état d'esprit de cette période, en proposant les principales variables à prendre en compte pour l'évaluation des projets agricoles pour le développement agricole en Afrique, en particulier. Egalement, d'autres auteurs ont abordé ces questions depuis la fin des années 90 en mobilisant les hypothèses théoriques qui structurent le cadre d'analyse du SIN en développant des guides méthodologiques sectoriels pour comprendre les systèmes d'innovation agricole (OCDE 2013). Le défis qui reste posé est d'élaborer un guide méthodologique de diagnostic des systèmes d'innovation agricoles en Afrique afin de reveller comment la connaissance peut devenir le principal facteur du développement socioéconomique. Le présent article analyse comment les anciennes approches de développement et l'application du SIN au secteur agricole permettent de proposer une méthodologie de diagnostic 
intégrée. Il s'inspire pour cela des travaux sur les Réseaux Techno-Economiques (Callon,1991), et contribue aux travaux plus globaux (Casadella et al.2006) à travers une documentation des experiences Africaines.

L'article est structuré comme suit. La première partie présente la démarche mobilisée. La deuxième partie fait l'état des approches sur l'innovation agricole en Afrique à partir de l'expérience du Burkina Faso. Enfin, la troisième partie fait une proposition méthodologique de diagnostic des systèmes d'innovation agricoles en Afrique.

\section{Méthodologie}

L'élaboration de la proposition méthodologique s'articule autour de deux stratégies dans le contexte d'étude du Burkina Faso. La première stratégie concerne une revue documentaire sur les approches linéaires de développement agricole.

Elle repose sur une description d'analyse d'études de cas concernant des interventions de développement agricole utilisant des approches linéaires. Elle décrit de manière concrète ces interventions et identifie de façon particulière leurs fondements théoriques. Ces approches sont abordées comme des « technologies », c'est-à-dire « des pratiques sociales et des relations sociales » (Sharif, 2004 :89). L'article propose d'examiner en même temps les transformations de ces technologies en relation avec les contextes de leurs utilisations. Cette exploration est complétée par l'analyse de la littérature concernant des travaux menés dans d'autres situations hors du Burkina Faso. Cette méthode vise donc de diagnostiquer les forces et les faiblesses de ces approches afin de les confronter aux approches interactionnelles, dans le processus d'élaboration de la méthodologie de diagnostic.

La deuxième strategie consiste à examiner des études de cas portant sur les approches interactionnelles de l'innovation agricole. Ces travaux reposent sur des cas ayant impliqué les auteurs du présent article. Ils reposent de ce fait sur près de dix années d'expériences pratiques dans l'accompagnement en développement rural et des travaux scientifiques sur le Système d'Innovation National (SIN), ainsi que son application dans le secteur agricole connu sous le nom de Système d'Innovation Agricole (SIA) au Burkina Faso. La première étude de cas de 2010, concerne le système d'innovation du coton biotechnologique au Burkina Faso qui a été explorée dans le cadre d'un Master en Sciences, Technologie et Société (Compaore 2010; Sawadogo-Compaore, 2018). Cette recherche a ainsi étudiée la structure et la dynamique des acteurs impliqués dans le système, en tant que moyen de comprendre le processus de traduction des nouvelles technologies dans le secteur du coton, et d'évaluer les résultats en termes d'avantages pour le développement économique et social pour les acteurs impliqués. La deuxième étude repose sur trois années de recherche doctorale (2011-2014) sur les conditions d'usage du modèle du Système d'Innovation National dans la planification du développement et sa traduction dans le secteur agricole burkinabé (Compaore 2015; Sawadogo-Compaore, 2017). L'objectif était d'étudier le rôle facilitateur du cadre des SIN dans la facilitation du développement socio-economique au Burkina Faso à travers deux niveaux de diffusion, le niveau politique (macro) et le niveau opérationnel (micro). Cette étude a élargi les recherches précédentes sur l'adoption et la mise en œuvre de l'innovation du coton Biotechnologique. En plus de ces deux études de cas, des recherches et des interventions supplémentaires sur des dimensions spécifiques du Système d'Innovation Agricole (SIA) ou sur le Système d'Innovation National (SIN) global ont été menées dans trois projets. En 2016, les deux auteurs ont participé à la mise en oeuvre du projet conjoint FAO-Gouvernement burkinabéAgrinatura sur le développement des capacités des systèmes d'innovation agricoles, qui visait à soutenir l'élaboration de politiques et d'approches dans les trois continents : Asie/Afrique/Amerique Latine (Compaoré-Sawadogo et al. 2017). Sur la période 2015-2018, l'un des auteurs a dirigé avec 
d'autres collègues (IDRC-DID-INERA et Université de Laval) un projet de trois ans (2015-2018) sur «la diffusion des services financiers et de l'innovation chez les populations rurales du Burkina Faso». La finance rurale y étant considérée comme un outil essentiel pour réduire la pauvreté. L'objectif était d'analyser le lien entre les ressources financières et la diffusion de l'innovation. Ce projet axé sur la recherche a ciblé les communautés agricoles (Pô, Sapouy, Gourci et Yako) et a évalué l'impact des services financiers communautaires traditionnels sur la production agricole, la sécurité alimentaire et la résilience, ainsi que sur les systèmes informels de prêts, d'emprunt et de crédit d'épargne (Sawadogo-Compaore 2017). Toutefois, il reste encore à démontrer comment mieux inclure les petits agriculteurs. En 2016 un des auteurs a eu le privilège de faire partie du Groupe de travail national sur l'évaluation des besoins technologiques agricoles, dans le cadre du programme stratégique mondial de Poznan sur le potentiel d'innovation technologique et l'adaptation au changement climatique. En 2017, un projet d'évaluation de l'écart de genre dans l'évaluation de la productivité agricole a été mené dans sept pays (Nigéria, Ghana, Burkina Faso, Mali, Uganda, Tanzanie, Kenya). Nous avons également explorée la question des relations entre l'innovation et le développement dans une contribution sur l'appropriation du développement centré sur le savoir en Afrique et de ses défis (Sawadogo et Sawadogo-Compaore, 2018). Cette série de recherches articule des travaux sur la compréhension théorique large du modèle de système d'innovation national avec des interventions concrètes plus spécifiques dans le système d'innovation agricole appliquant le modèle SIN au secteur agricole. Elle nourrit à la fois une maitrise théorique sur les expériences, sur les propriétés et la dynamique du système d'innovation agricole du Burkina Faso mais explore aussi ponctuellement d'autres situations en Afrique. Elle conduit à mobiliser cette expérience pour proposer une méthodologie de diagnostic des systèmes d'innovation agricoles adaptée au contexte géographique spécifié. Le cadre méthodologique proposé ici ne se veut pas universel. Son objectif modeste est de contribuer à la connaissance du système d'innovation agricole du Burkina Faso, en particulier, et de ceux des pays africains, qui partagent plus ou moins les mêmes caractéristiques structurelles. Il invite ainsi à des réflexions en collaboration avec d'autres collègues africains intéressés par les mêmes questions; et pourquoi pas des partenariats de recherches plus globaux dans le but de développer une méthodologie plus robuste pour le diagnostic des SIA en Afrique. L'ensemble de ces études de cas, travaux, interventions sont analysés du point de vue de leurs forces et faiblesses dans leurs capacités à activer les modèles interactionnels dans la réalisation de l'innovation agricole. Cet ensemble repose sur les connaissances acquises parfois dans des études de cas qui se referrent aux modèles linéaires et dans le cadres des SIN. L'objectif, bien que contextualisé à un seul pays, est d'élaborer une démarche méthodologique basée sur des preuves avec l'ambition de constituer un cadre de référence plus global pour d'autres chercheurs.

\section{Modèle Linéaire de l'innovation agricole}

Au Burkina Faso, «l'approche intégrée autour des cultures de rente» constitue la toute première approche de transfert de technologies agricoles. La période de 1900 aux années 1970 est marquée par ce que l'on appelle le transfert technologique des pays développés vers les pays sous-développés (Gaillard 1999). Cette approche intégrée en tant qu'outil de diffusion de l'innovation consiste en un processus qui s'étend de la production à la transformation et à la commercialisation des produits dérivés sur le marché. Dans le secteur agricole, dans lequel cet outil a été principalement appliqué, l'intégration verticale donne plus de pouvoir aux industries en leur donnant le rôle de médiateurs de l'innovation ainsi que celui de fournisseurs d'intrants et de crédits, etc. Elles fournissent aux agriculteurs tout ce dont ils ont besoin pour leurs cultures de rente, ces derniers devant leurs rembourser après leurs récoltes. Les instituts de recherche, qui mènent des recherches pour résoudre les difficultés liées à ces cultures de rente, n'interagissent alors qu'avec ces industries et non avec les agriculteurs (Belloncle 1987). Les chercheurs dans ce processus linéaire sont informés des besoins des agriculteurs par les agents d'appui technique employés par les industries. Il n'y a pas de contact direct et simultané entre les agents de l'agriculture, les agriculteurs et les chercheurs (Evanson 
1987). Le chercheur étant dans son monde d'expert laissant le producteurs entre les mains de l'agent technique de l'agriculture qui est l'intermediaire entre les chercheurs et les producteurs. C'est une approche avec un modèle de communication descendant, laissant peu de place à la participation des differents acteurs. Le tableau suivant donne les caractéristiques de cette approche.

\begin{tabular}{|l|l|l|l|l|l|l|l|l|l|l|}
\hline & \multicolumn{3}{|c|}{ Composantes } & \multicolumn{3}{|l|}{$\begin{array}{l}\text { Potentiel de } \\
\text { participation }\end{array}$} & \multicolumn{3}{|c|}{$\begin{array}{l}\text { Création de richesses à } \\
\text { partir des connaissances }\end{array}$} \\
\hline $\begin{array}{l}\text { approche de } \\
\text { diffusion } \\
\text { d'innovation }\end{array}$ & $\begin{array}{l}\text { Rôle du du } \\
\text { Government }\end{array}$ & $\begin{array}{l}\text { Rôle des } \\
\text { universités }\end{array}$ & $\begin{array}{l}\text { Rôle des } \\
\text { industries }\end{array}$ & & $\mathbf{1}$ & $\mathbf{2}$ & $\mathbf{3}$ & $\mathbf{4}$ & $\mathbf{5}$ & $\mathbf{6}$ \\
\hline $\begin{array}{l}\text { Top-Down ou } \\
\text { descendante }\end{array}$ & & & $\mathrm{X}$ & $\begin{array}{l}\text { Sans } \\
\text { Participation }\end{array}$ & & $\mathrm{X}$ & & & & \\
\hline
\end{tabular}

Tableau 1. Descriptif de l'approche linéaire de l'innovation agricole

Au plan théorique, les bases conceptuelles d'un tel modèle sont connues. En effet, prédominante dans les années 1950, 1960 et au début des années 1970, c'est à travers les théories linéaires diffusionistes que le processus de croissance économique a été étudié. Ces théories considéraient généralement l'innovation comme dépendant de découvertes scientifiques. Selon Godin (2009), le premier modèle s'appelait Science-Push (Godin 2009). Il a été ensuite suivi du modèle appelé Demand-Pull. Dans le premier il est assumé que toute découverte scientifique conduit nécessairement à une innovation technologique à travers son utilisation par des «usagers de la connaissance », particulièrement les entreprises. La même posture est adoptée dans le second qui atribue cependant à la mise au point de la découverte, à la formation de la demande et au développement du marché (Manley 2003; Godin 2009).

\section{Modèles Interactionnels de l'Innovation Agricole}

Vers la fin des années 1970, le peu de satisfaction des expériences en matières de développement remet en question les modèles précedents et commence à transformer les pratiques de développement. C'est ainsi que des approches de plus en plus interactionnelles vont donc se développer.

\subsection{Approche participative du développement agricole ou approche "formations et visites "}

L'approche participative de la diffusion de l'innovation, baptisée Top-Down Bottom-Up en anglais, a été développée par Daniel Benor pour le compte de la Banque mondiale dans les années 1970 (Russell, 1987). Elle consiste à organiser des visites d'agriculteurs effectuées par des agents techniques à un moment qui convient à l'agent et à l'agriculteur. Une personne qui a vecu pendant cette période décrit cette approche en ces termes:

\footnotetext{
${ }^{1}$ 1- Les entrepreneurs qui tentent de créer de nouvelles entreprises innovantes qui déploient leurs connaissances de manière novatrice. 2- Les dirigeants d'entreprises qui cherchent à améliorer leur productivité grâce à l'application efficace de nouvelles connaissances et soucieux de la «réinvention» de l'entreprise par le biais d'une forme d'entrepreneuriat; 3-Les responsables des structures publiques 3-R \& D des secteurs publics et privés tentent d'améliorer l'utilisation des connaissances créées au sein de leurs groupes; 4-Fournisseurs de services métier à forte intensité de connaissances essayant de créer une entreprise en permettant des connexions et des flux au sein du système de connaissances; 5-Les agents des politiques gouvernementales aux niveaux local, régional et national chargés d'améliorer l'efficacité et l'efficience du système d'innovation; 6-Les 'propriétaires' de chaînes d'approvisionnement désireux d'améliorer l'efficience/efficacité de leurs réseaux au niveau du système par l'innovation.
} 
à la fin des années 1980, un consultant de la Banque mondiale, Benor, développa un système appelé TV (Training and Visit). Mais c'était toujours le même processus linéaire où entre chercheurs et agriculteurs, il n'y avait pas de communication. La relation est entre chercheurs et agents techniques qui se rencontrent toujours. L'agriculteur est tenu à l'écart, seul l'agent technique peut rencontrer les chercheurs. La pensée linéaire existe donc toujours, mais il y a eu un dialogue entre agents techniques et chercheurs lors de réunions et un dialogue entre ces agents techniques et les agriculteurs [...] (entretien $\mathrm{n}^{\circ}$ 16).

Ainsi donc, c'est une approche toujours orientée sur le producteur, mais avec un modèle communicationnel descendant-ascendant, laissant plus de place à la participation des producteurs. Le tableau suivant donne les caractéristiques de l'approche.

\begin{tabular}{|l|l|l|l|l|l|l|l|l|l|l|}
\hline & \multicolumn{3}{|c|}{ Composantes } & \multicolumn{2}{|l|}{$\begin{array}{l}\text { Potentiel de } \\
\text { participation }\end{array}$} & \multicolumn{3}{|c|}{$\begin{array}{l}\text { Création de richesses à } \\
\text { partir des connaissances }\end{array}$} \\
\hline $\begin{array}{l}\text { approche de diffusion } \\
\text { d'innovation }\end{array}$ & $\begin{array}{l}\text { Rôle du } \\
\text { Government }\end{array}$ & $\begin{array}{l}\text { Rôle des } \\
\text { universités }\end{array}$ & $\begin{array}{l}\text { Rôle des } \\
\text { industries }\end{array}$ & & 1 & 2 & 3 & 4 & 5 & 6 \\
\hline $\begin{array}{l}\text { Top-Down Bottom-Up } \\
\text { (Formation et Visite) }\end{array}$ & & & $x$ & $\begin{array}{l}\text { Participation } \\
\text { limitée }\end{array}$ & & $x$ & & & \\
\hline
\end{tabular}

Tableau 2. Descriptif de l'approche formation et visite

\section{2. À la recherche d'une approche de diffusion d'innovation interactionelle}

Il s'agit de l'approche système agricole. Dans cette approche interactionnelle, l'objectif est que «des groupes spécifiques partageant des besoins et vivant dans une zone géographique définie recherchent activement l'identification de leurs besoins, prennent des décisions et établissent des mécanismes pour répondre à leurs besoins» (Rifkin et al., 1988), cités par (Bjaras, Haglund et Rifkin, 1991: 2000). C'est une approche dont l'orientation est stratégique et va au-delà du producteur avec un modèle communicationnel interactionnel, permettant à tous les acteurs d'interagir les uns avec les autres directement. Le tableau suivant donne les caractéristiques de l'approche.

\begin{tabular}{|c|c|c|c|c|c|c|c|c|c|c|}
\hline & \multicolumn{3}{|c|}{ Composantes } & \multirow[t]{2}{*}{$\begin{array}{l}\text { Potentiel de } \\
\text { participation }\end{array}$} & \multicolumn{6}{|c|}{$\begin{array}{l}\text { Création de richesses à } \\
\text { partir des connaissances }{ }^{3}\end{array}$} \\
\hline $\begin{array}{l}\text { Approche de } \\
\text { diffusion } \\
\text { d'innovation }\end{array}$ & $\begin{array}{l}\text { Rôle du } \\
\text { Gouvernement }\end{array}$ & $\begin{array}{l}\text { Rôle des } \\
\text { universités }\end{array}$ & $\begin{array}{l}\text { Rôle des } \\
\text { industries }\end{array}$ & & 1 & 2 & 3 & 4 & 5 & 6 \\
\hline $\begin{array}{l}\text { Système agraire } \\
\text { ou agricole }\end{array}$ & & & $x$ & $\begin{array}{l}\text { Participation } \\
\text { limitée }\end{array}$ & & $x$ & & $x$ & & \\
\hline
\end{tabular}

Tableau 3. Descriptif de l'approche système agricole

En 2005, deux expériences impliquant des approches interactionnelles émergent. L'une repose sur le cadre d'analyse des SIN qui est l'objet de cet article, et l'autre sur la mise en place de

\footnotetext{
${ }^{2}$ Idem page 5

${ }^{3}$ Idem page 5

Technologie et Innovation, 2020, vol. 5, $n^{\circ} 3$

(C) 2020 ISTE OpenScience - Published by ISTE Ltd. London, UK - openscience.fr
} 
Plateformes Multi-acteurs (PMA). Selon le Ministère de la Recherche et de l'Innovation du Burkina (2012: 11), la PMA est «un regroupement de moyens (essentiellement matériels et humains) pour une communauté ouverte spécifique où les utilisateurs, principalement les entreprises, sont en interaction (localisation des équipements, expérimentations de services), leur permettant de mener leurs projets d'innovation en matière de recherche et développement ( $\mathrm{R} \& \mathrm{D})$ ».

\begin{tabular}{|c|c|c|c|c|c|c|c|c|c|c|}
\hline \multirow[b]{2}{*}{$\begin{array}{l}\text { approche de } \\
\text { diffusion } \\
\text { d'innovation }\end{array}$} & \multicolumn{3}{|c|}{ Composantes } & \multirow[t]{2}{*}{$\begin{array}{l}\text { Potentiel de } \\
\text { participation }\end{array}$} & \multicolumn{6}{|c|}{$\begin{array}{l}\text { Création de richesses à } \\
\text { partir des connaissances }\end{array}$} \\
\hline & $\begin{array}{l}\text { Rôle du } \\
\text { Gouvernement }\end{array}$ & $\begin{array}{l}\text { Rôle des } \\
\text { université }\end{array}$ & $\begin{array}{l}\text { Rôle des } \\
\text { industries }\end{array}$ & & 1 & 2 & 3 & 4 & 5 & 6 \\
\hline $\begin{array}{l}\text { Plateforme Multi- } \\
\text { acteurs }\end{array}$ & & & $X$ & $\begin{array}{l}\text { Participation } \\
\text { renforcée }\end{array}$ & & $x$ & $\mathrm{x}$ & $\mathrm{x}$ & & $x$ \\
\hline
\end{tabular}

Tableau 4. Descriptif de l'approche plateforme multi-acteurs de l'innovation agricole

Depuis la publication phare de l'OCDE sur la gestion des systèmes nationaux d'innovation en 1999, le cadre appelé Systèmes d'Innovation National (SIN) est de plus en plus populaire. Il a d'abord été développé sous forme de cadre théorique, de concept et de perspective analytique puis utilisé pour étudier le flux de technologies et d'informations entre les individus, les entreprises et les institutions. Ce réferentiel d'analyse systémique diverge des modèles précédents caractérisés de linéaires.

\subsection{Description du cadre des systèmes d'innovation nationaux}

\section{Origine et travaux pionniers}

L'usage de l'analyse systémique appliqué à l'innovation est à l'orgine de la conception des SINs qui structure des modalités d'interdépendances entre les sciences fondamentales, du marketing, de la fabrication et de la Recherche et le Développement (R\&D) (Manley, 2003; Godin, 2009). L'innovation y est considérée comme un processus interactif et complexe. Le concept de systèmes d'innovation national a été développé dans les années 1980 par Freeman (1982) et Lundvall (1985) pour expliquer comment l'innovation technologique résulte de l'interaction complexe entre institutions (Lundvall, 2007). Ce cadre conceptuel a ensuite été adopté dans les études sur la science, la technologie et l'innovation à partir des années 1990 pour étudier les systèmes d'innovation (Nelson, 1993; Freeman, 1995; Lundvall, 1992; Lundvall, 2007). Godin (2009) a montré que «l'approche systémique» mise au point par l'OCDE s'appuie en grande partie sur les travaux de recherche sus-cités depuis les années 1960.

\section{Objectifs/fonctions d'un SIN}

Selon Lundvall, un SIN a pour objectif de réaliser la compétitivité internationale des économies nationales, et de conduire à la croissance de l'économie nationale. Pour lui, il semble y avoir une acceptation sociale plus large du concept de «national ». Mais ce n'est pas le seul niveau pertinent à prendre en compte (Lundvall, 2007). Il s'en suit que la dimension sociale reste incertaine à réaliser dans le cadre national. C'est plutôt à travers les organisations internationales (continentales) d'une part et celles mondiales (ex. Agences des Nations Unies, Organisations environnementalistes mondiales) d'autres part, que cette dimension pourrait être réalisée. Concernant les organisations

\footnotetext{
${ }^{4}$ Idem page 5 
internationales, les politiciens et les experts de ce niveau sont davantage orientés vers le renforcement de la croissance économique de leurs régions respectives et vers la prévention des conflits internationaux au sein de la communauté des pays qu'ils représentent. (Lundvall, 2007). Dans le cas des organisations mondiales il devient plus évident pour les experts et les acteurs politiques que la survie à long terme de l'économie mondiale dépend de la durabilité écologique et d'une réduction de l'inégalité sociale extrême au niveau mondial (Lundvall, 2007). En clair, le SIN reste limité quand il s'agit de promouvoir ou prendre en compte la dimension sociale du développement.

\section{La nature des processus d'innovation complexes et interactifs}

Un système d'innovation national, suppose des producteurs de connaissances, en l'occurrence les universités. Ces dernières sont essentielles à la création ou à l'invention de toute innovation, car la performance $\mathrm{du}$ système dépend $\mathrm{du}$ capital humain et des capacités. Ces producteurs de connaissances sont produits par des universités, des organisations techniques et des écoles, par exemple (Lundvall, 2007). Les universités en particulier ont leurs propres unités de recherches qui facilitent la formation du marché en fournissant de nouvelles connaissances et compétences et en menant des recherches (Lundvall, 1985; 2007; Freeman, 1987; OCDE, 1999). Ces instituts de production du savoir sont des centres de recherches fondamentales et de formations scientifiques (Lundvall, 1985; OCDE, 1999). En d'autres termes, les universités en tant que producteurs de connaissances s'engagent à cet égard dans les sciences fondamentales et la recherche technologique. Dans le même temps, les universités proposent aux scientifiques et aux technologues un enseignement supérieur qui leur permet de générer l'innovation dont les entreprises et les gouvernements ont besoin. Nelson a également souligné ce rôle des universités: «[Les universités offrent] des niveaux de formation scientifique et des compétences de gestion plus élevés pour coordonner ce qui est inévitablement une affaire à plusieurs personnes ou à plusieurs entreprises» (Nelson, 1993, Feinson, 2003: 24). Toutefois, dans le modèle des SIN, cela n'est pas suffisant, d'autres acteurs sont nécessaires.

Le deuxième acteur clé sont les entreprises. Elles sont toutes considérées comme des acteurs économiques qui utilisent l'innovation créée par les producteurs de connaissances comme les universités et des centres de recherches (OCDE, 1999). Dans les SIN, les entreprises contribuent à la diffusion de la connaissance sur le marché (OCDE, 1999). Lundvall (1992), Nelson (1993) et Edquist (1997) ont examiné l'idée d'un système dans lequel les entreprises sont en interaction avec les deux variables susmentionnées. Comme l'a démontré l'OCDE (1999), les entreprises ou l'industrie ne fonctionnent pas seules, car les pouvoirs publics, tout comme les universités, font également partie intégrante du système dans le processus d'innovation. Les entreprises, quant à elles, sont considérées comme des acteurs économiques dont le rôle est de mener des activités de R\&D afin de développer des produits destinés à la commercialisation. Ils lancent les différents produits innovants et exploitent les nouvelles découvertes scientifiques (OCDE, 1999). Pour les auteurs du cadre des SIN, se limiter aux entreprises signifie rester dans le modèle de « demandpull ». Aussi bien les entreprises que les universités ne peuvent adequatement travailler que s'il existe un environnement favorable. En effet, de nombreux spécialistes du système d'innovation ont explicitement expliqué le fait que le gouvernement devrait participer activement (Freeman, 1995) à la promotion de l'innovation. Au cours des années 1990 et 2000, l'importance du système d'innovation par rapport aux nouvelles technologies a été largement acceptée et son utilisation a commencé à se répandre dans les pays développés et en développement (OCDE, 1999). En outre, l'importance de la connaissance dans le processus de diffusion de l'innovation fait l'objet d'une attention particulière. En conséquence, la connaissance a été identifiée comme un moteur (1999) pour atteindre et renforcer une croissance économique durable. Cette vision contemporaine a été largement adoptée et a eu un impact significatif sur la stratégie et la politique d'innovation, en particulier dans les pays développés. Les contributions des institutions individuelles ne sont pas 
considérées isolément mais avec «la manière dont elles interagissent les unes avec les autres en tant qu'éléments d'un système collectif de création et d'utilisation des connaissances, et leur interaction avec les institutions sociales : valeurs, normes, cadres juridiques, etc. (Smith in OECD, 1999: 24). Dans cette interaction, le gouvernement peut jouer un rôle clé par le biais de ses options politiques et de ses réglementations. Les approches des systèmes d'innovation, étayées par des hypothèses et des conventions d'encadrement fondées sur les expériences des pays développés, ont été appliquées dans les pays en développement sans trop de modifications par rapport aux différents contextes.

\section{L'importance des flux de connaissances et de technologies}

De manière générale, la théorie des systèmes nationaux d'innovation souligne que les flux de technologie, et d'informations entre les individus, les entreprises et les institutions sont essentiels au processus d'innovation. Ainsi, l'innovation et le développement technologique résultent d'un ensemble complexe de relations entre les acteurs du système, qui comprend les entreprises, universités et instituts de recherche gouvernementaux (Godin, 2009). L'inclusion du système de recherche du pays dans cet ensemble complexe de relations est un autre élément distinctif de cette approche. Godin note que :

le cadre du système d'innovation national [théorie] suggère que le but ultime du système de recherche est l'innovation et que le système fait partie d'un système plus vaste composé de secteurs tels que le gouvernement, les universités, l'industrie et leur environnement. Le cadre a également mis l'accent sur les relations entre les composants ou les secteurs, en tant que «cause» expliquant la performance des systèmes d'innovation. (Godin 2009: 476-477).

Cette théorie pose pour postulat que la compréhension des liens entre les acteurs impliqués dans l'innovation est la clé de l'amélioration des performances technologiques. L'innovation et le progrès technique résultent d'un ensemble complexe de relations entre acteurs produisant, diffusant et appliquant divers types de connaissances (OCDE, 1999). On y voit une nouvelle façon de faire ou une nouvelle conception d'un produit existant pour un marché de « niche » ou une modification de la manière dont le travail est effectué dans une organisation (OCDE, 1997).

\section{Rôle des institutions et des interactions entre institutions}

Le concept de SIN concerne également deux catégories principales - les institutions de marché et les institutions non marchandes (Manley, 2003). Les deux sont considérées pour leur potentiel d'influence sur l'orientation et la vitesse de l'innovation et la diffusion de la technologie dans un pays (OCDE, 1999). Il est important de noter que cette théorie a été développée principalement à partir de l'étude des systèmes d'innovation des pays développés (Freeman 1987; OCDE, 1999). À cet égard, comme le dit Sharif, «le système d'innovation national est considéré comme l'ensemble des institutions et des pratiques qui interagissent pour produire et diffuser de nouvelles technologies» (Sharif 2006: 84). Ici, le SIN est considéré comme un objet d'étude au sens d'appellation de SIN britannique, SIN des Etats-Unis, SIN du Burkina Faso, etc., pour désigner un Système que l'on peut étudier en tant qu'objet ou transformer en tant que matière. Comme le dit Freeman, «... le réseau d'institutions des secteurs public et privé dont les activités et les interactions initient, importent, modifient et diffusent les nouvelles technologies» (Freeman, 1987 dans OCDE, 1999: 10).

\section{Rôle du gouvernement et du cadre de politiques nationales et de l'État-nation}

L'acquisition de la technologie, son utilisation et sa diffusion impliquent le gouvernement, dont le rôle dans le système est essentiel. Par le biais de ses décideurs, le gouvernement joue un rôle clé en faisant des choix qui éclairent les stratégies au niveau national (Freeman, 1987). En effet, le gouvernement est considéré comme un acteur principal influant d'une part sur les Investissements 
Directs Etrangers (IDE) car, «malgré leur taille limitée, les entrées d'IDE ont eu un impact positif, tout en générant des emplois dans le secteur formel et génération de valeur ajoutée locale »(Nations Unies, 2009: 2). D'autre part, un tel flux est pris en charge dans le cadre de la réglementation gouvernementale en matière de DPI (Droit de la Propriété Intellectuelle) dans laquelle les processus sont décomposés en trois étapes: processus d'innovation, processus de commercialisation et processus de protection des DPI. En concevant le système des DPI dans l'intérêt de la recherche scientifique directe des entreprises et des pouvoirs publics, comme le financement des universités, le soutien à la recherche et au développement des entreprises (R \& D), «il incombe au gouvernement de contribuer à la formation du capital humain et social nécessaire pour évaluer, choisir, mettre en œuvre et modifier les technologies étrangères» (Feinson, 2003: 23). La R \& D peut être utilisée pour l'assimilation des connaissances et la création technologique nationale, comme le montre Freeman dans son étude de cas sur le Japon (Freeman, 1987). Les incitations créées par le gouvernement sont nécessaires pour définir ces aspects au niveau de l'orientation de la politique nationale afin de fournir un soutien administratif ainsi que des installations et du matériel permettant de renforcer et de légitimer la mise en réseau des producteurs et des utilisateurs de connaissances (Lundvall, 1985; Freeman, 1997 ; OCDE, 1999). L'engagement du gouvernement ainsi que des actions spécifiques visant à fournir aux acteurs un tel contexte sont donc nécessaires pour créer un contexte social et politique favorable à la mise en réseau des entreprises et des producteurs de connaissances. Dans ce modèle, les acteurs pertinents et leur rôle sont plus connus, et les éffets de leurs combinaisons maîtrisés. Cependant bien que les acteurs soient en interaction, le traitement économetrique du modèle ne permet pas de comprendre les processus à travers lesquels les acteurs interagissent. L'innovation est pourtant un processus social qui a besoin du mouvement des acteurs dont les paramettres doivent êtres pris en compte dans toute analyse.

\section{Le SIN, comme outil d'analyse stratégique et de diagnostic}

Depuis la vulgarisation du concept par l'OCDE, les SIN sont utilisés comme outil politique ou modèle pour orienter les systèmes d'innovation au niveau national. L'intérêt du gouvernement pour les outils politiques des SIN est particulièrement utile pour cet article, car il renvoie à une troisième définition du cadre dans laquelle, en ce qui concerne le cercle des politiques, les SIN sont considérés comme un «outil politique» (Lundvall 2007: 1). Ce concept devient alors un outil de pilotage pour donner une cohérence pratique aux décideurs afin de concevoir et mettre en œuvre leurs politiques. Il peut ainsi être considéré comme un récit de politique à travers lequel les décideurs élaborent leurs politiques. Godin saisit clairement cet aspect lorsqu'il dit que «les décideurs élaborent leur problème à travers des cadres conceptuels qui structurent l'action politique» (Godin 2009: 5).

C'est à cet égard que les SIN peuvent être considérés comme un modèle, c'est-à-dire «une technologie qui nous permet de [...] faire en sorte que les choses fonctionnent dans le monde» (Morrison et Morgan 1999: 32). Dans le cadre de cet article, c'est cette dimension (utilisation du cadre des SIN comme outil politique au Burkina Faso et stratégie de développement sectoriel) qui est prise en compte. Dans le cas spécifique du Burkina Faso, un projet a été ainsi initié en 2006 par le FRSIT (représentant le gouvernement) et le CRDI, dans le but d'introduire le cadre du SIN comme un outil de politique afin d'améliorer ce que l'on pourrait appeler le SIN du Burkina Faso. 


\begin{tabular}{|c|c|c|c|c|c|c|c|c|c|c|}
\hline \multirow[b]{2}{*}{$\begin{array}{l}\text { Approche de } \\
\text { diffusion } \\
\text { d'innovation }\end{array}$} & \multicolumn{3}{|c|}{ Composantes } & \multirow[t]{2}{*}{$\begin{array}{l}\text { Potentiel de } \\
\text { participation }\end{array}$} & \multicolumn{6}{|c|}{$\begin{array}{l}\text { Création de richesses à partir des } \\
\text { connaissances }{ }^{1}\end{array}$} \\
\hline & $\begin{array}{c}\text { Rôle du } \\
\text { Gouvern } \\
\text { ement }\end{array}$ & $\begin{array}{l}\text { Rôle des } \\
\text { universités }\end{array}$ & $\begin{array}{l}\text { Rôle des } \\
\text { industries }\end{array}$ & & 1 & 2 & 3 & 4 & 5 & 6 \\
\hline $\begin{array}{c}\text { Système } \\
\text { d'Innovation } \\
\text { National }\end{array}$ & $x$ & $X$ & $X$ & $\begin{array}{c}\text { méthodes } \\
\text { Participatives mais } \\
\text { pas adaptée à tous les } \\
\text { contextes surtout } \\
\text { dans les pays en } \\
\text { développement }\end{array}$ & $x$ & $x$ & $x$ & $x$ & $x$ & $X$ \\
\hline
\end{tabular}

Tableau 5. Descriptif de l'approche SIN

\section{Au-delà du SIN : prendre le contexte comme un aspect essentiel}

La perspective d'analyse sociologique que développe l'école française d'innovation, en termes de « Réseau Techno-Economique » (RTE), fournit un modèle qui complémente les insuffisances relevées dans les différentes approches décrites ci-dessus. Son approche moins positiviste permet des possibilités d'adaptation des modalités de communication dans les SIN. En effet, selon Callon et ses collaborateurs, un Réseau Techno-Economique (RTE) est « un ensemble coordonné d'acteurs hétérogènes (laboratoires publics, centres de recherches technologique, entreprises industrielles, utilisateurs) qui participent collectivement au développement et à la diffusion de l'innovation et, au moyen de nombreuses interactions, organisent les relations entre la recherche scientifique et technologique et le marché » (Callon and al. 1992 :215). Ce modèle permet d'intégrer les forces des approches précédentes, dont la pertinence a été finalisée dans le contexte des pays industriels. Sur le plan communicationnel, les outils de communication avec une population majoritairement analphabète, se sont stabilisés. C'est ce qui manque au modèle du SIN, qui porte en fait les caractères de son contexte d'élaboration, qui est celui des pays développés (Katz et al.,2014). La méthodologie pratique de diagnostic des systèmes d'innovation agricoles proposée prend en compte les forces et les faiblesses des principales approches d'innovation agricoles jusque-là utilisées au Burkina Faso, et en Afrique en général.

\section{Méthodologie pratique pour le diagnostic de systèmes d'innovation agricole en Afrique}

Sur la base des développements ci-dessus, nous identifions six (6) étapes clés pertinentes pour le diagnostic des systèmes d'innovation agricole en Afrique.

Étape 1: Comprendre les principales caractéristiques d'un système d'innovation agricole: décrire les principales caractéristiques d'un système d'innovation agricole

\begin{tabular}{|l|l|}
\hline \multicolumn{1}{|c|}{ Variables } & $\begin{array}{c}\text { Techniques de } \\
\text { collecte des } \\
\text { données }\end{array}$ \\
\hline$\checkmark$ & $\begin{array}{l}\text { Diversité des secteurs (industrie agricole, systèmes de recherche } \\
\text { et d'enseignement en agriculture, agences gouvernementales en }\end{array}$ \\
$\begin{array}{l}\text { agriculture) } \\
\checkmark\end{array}$ & $\begin{array}{l}\text { Quantitatives et } \\
\text { qualitatives } \\
\text { recherche des et d'enseignement, agr. Institutions financières, }\end{array}$ \\
\hline
\end{tabular}


travailleurs agricoles, normes)

$\checkmark$ Variété d'acteurs (différentes catégories d'entreprises agricoles, différentes catégories d'institutions de recherche et d'enseignement, différentes catégories d'institutions financières, différentes catégories socioprofessionnelles, normes)

$\checkmark$ Existence de liens entre les secteurs et entre les acteurs

$\checkmark$ Existence d'interactions entre les secteurs et entre les acteurs

$\checkmark$ Complémentarité entre les secteurs et entre les acteurs

$\checkmark$ Dynamique - il évolue dans le temps et en fonction des enjeux

$\checkmark$ Décrire les principales fonctions d'un système d'innovation agricole efficace et efficient

$\checkmark$ Permettre le flux de connaissances

$\checkmark$ Facilitation (soutien: institutionnel, financier, compétence, etc.)

$\checkmark$ La communication

$\checkmark$ Action collective

$\checkmark$ Générer de l'innovation

$\checkmark$ Il établit des normes pour une performance efficace et efficiente

Étape 2: Identifier les éléments clés d'un système d'innovation agricole. Quels types de groupes, de réseaux, faudrait-il mettre en place pour créer un SIA efficace (optimal souhaité)?

\begin{tabular}{|c|c|}
\hline Variables & $\begin{array}{l}\text { Techniques de } \\
\text { collecte des données }\end{array}$ \\
\hline $\begin{array}{l}\text { Les données à collecter I: consistent en une analyse technico- } \\
\text { économique et comprennent: premièrement, l'identification des } \\
\text { acteurs, notamment i) les laboratoires publics ii) les centres de } \\
\text { recherche technique iii) les entreprises industrielles iv) les } \\
\text { organisations financières v) les utilisateurs vi) les pouvoirs publics. } \\
\text { Deuxièmement, la caractérisation de ces acteurs du réseau techno- } \\
\text { économique en fonction de leurs pôles correspondants: i) Pôle } \\
\text { scientifique (S), se référant à la production de connaissances } \\
\text { scientifiques certifiées (articles de revues / laboratoires), de } \\
\text { rapports, de notes de travail (à diffusion limitée, même semi- } \\
\text { automatique) -confidentiel), instruments (objets techniques), } \\
\text { compétences incorporées (chercheurs formés dans des universités } \\
\text { en transition vers le secteur de l'industrie / du développement } \\
\text { considéré). ii) Pôle technologique (T) consistant en l'identification } \\
\text { d'objets matériels conçus ayant une cohérence propre. iii) Pôle de } \\
\text { marché (M) consistant en l'identification de réseaux de distribution } \\
\text { et de commercialisation d'organisations (privé, public; commercial } \\
\text { / non commercial), ainsi que en tant qu'utilisateurs ou groupes de } \\
\text { consommateurs liés aux organisations de distribution et de } \\
\text { redistribution. }\end{array}$ & $\begin{array}{l}\text { Techniques de } \\
\text { collecte de données: } \\
\text { les techniques de } \\
\text { collecte de données } \\
\text { comprennent le } \\
\text { questionnaire, la } \\
\text { revue documentaire, } \\
\text { y compris les } \\
\text { archives, les } \\
\text { interviews et } \\
\text { l'observation directe. } \\
\text { Les données seront } \\
\text { analysées à l'aide de } \\
\text { techniques } \\
\text { bibliométriques et } \\
\text { scientométriques, } \\
\text { ainsi que d'analyses } \\
\text { de contenu. }\end{array}$ \\
\hline $\begin{array}{l}\text { Pour interagir et justifier les niveaux d'interaction, d'autres acteurs } \\
\text { sont nécessaires: les intermédiaires. } \\
\text { Données à collecter II: collecte de documents écrits (articles } \\
\text { scientifiques, rapports, brevets, etc.), compétences incorporées }\end{array}$ & \\
\hline
\end{tabular}


(chercheurs changeant de laboratoires, ingénieurs passant d'une entreprise à une autre, etc.),(financements ou source de revenus) (contrats de coopération, prêts financiers, achat par clients de biens ou de services, etc.), objets plus ou moins techniques (prototypes, machines, produits destinés à la consommation financière, etc., sites de test, normes, règles de l'art, méthodes). Celles-ci seront acheminées selon les deux pôles intermédiaires: pôle de transfert (ST) pour les organisations d'interactions scientifiques et technologiques et pôle de développement (TM) pour les activités de production et de distribution entre technologie et marché (ressources technologiques et marché).

Étape 3: Évaluation du système d'innovation agricole existant

Question de recherche: Quels sont les objectifs stratégiques du système national d'innovation agricole?

\begin{tabular}{|c|c|}
\hline Variables & $\begin{array}{l}\begin{array}{l}\text { Techniques de collecte des } \\
\text { données }\end{array} \\
\end{array}$ \\
\hline $\begin{array}{l}\text { Données à collecter: Il s'agira tout d'abord } \\
\text { d'identifier les missions, consistant à : } \\
\text { i) élucider les principes généraux d'action / } \\
\text { politique. } \\
\text { ii) de définir le cadre d'action / politique. } \\
\text { Deuxièmement, il s'agira d'identifier les } \\
\text { «produits» stratégiques concernés par la } \\
\text { politique nationale sur les SIA notamment } \\
\text { a) les listes de produits ou de familles de } \\
\text { produits à développer comme stratégiques pour } \\
\text { les missions politiques, } \\
\text { b) les domaines de développement liés à ces } \\
\text { produits considérés comme stratégiques pour la } \\
\text { mission politique. }\end{array}$ & $\begin{array}{l}\text { Techniques de collecte de données: une } \\
\text { analyse documentaire et des entretiens } \\
\text { seront utilisés pour la collecte de } \\
\text { données. L'analyse du contenu et } \\
\text { l'analyse de mots associés seront } \\
\text { utilisées. } \\
\text { Résultats de l'analyse: identification des } \\
\text { critères utilisés pour les options } \\
\text { stratégiques; listes de produits, ou } \\
\text { familles de produits identifiés par la } \\
\text { politique, et justification associée. }\end{array}$ \\
\hline
\end{tabular}

Étape 4: Évaluer le modèle appliqué / mis en auvre pour le développement d'un système d'innovation agricole

Cette étape évalue les bases épistémologiques et théoriques sous-jacentes des interventions sur le système d'innovation agricole national. Il s'agit de répondre à la question suivante : l'innovation agricole considérée s'inscrit-elle dans une approche linéaire de diffusion d'innovation ou bien interactionnelle? laquelle?

Étape 5: Comparaison du système d'innovation agricole existant et du système d'innovation agricole optimal souhaité (standard)

Question de recherche: Qu'est-ce qui a été réalisé depuis?

\begin{tabular}{|l|l|}
\hline \multicolumn{1}{|c|}{ Variables } & $\begin{array}{l}\text { Techniques de } \\
\text { collecte des } \\
\text { données }\end{array}$ \\
\hline $\begin{array}{l}\text { Données à collecter: Modèles de réseaux techno-économiques à } \\
\text { travers des descriptions et une analyse comparative souhaitées par }\end{array}$ & $\begin{array}{l}\text { Quantitatives et } \\
\text { qualitatives }\end{array}$ \\
\hline
\end{tabular}


rapport aux SIA existants fournis par l'évaluation technoéconomique. Cela permettra d'identifier les configurations que le réseau technico-économique peut adopter tout au long de la mise en œuvre des interventions SIA.

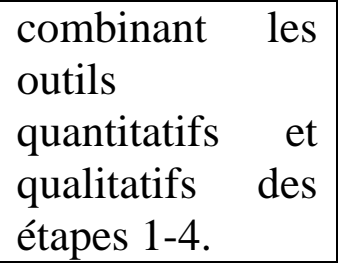

Étape 6: Interprétation du portefeuille du système d'innovation agricole (extrants / résultats)

\begin{tabular}{|c|c|}
\hline Variables & $\begin{array}{l}\text { Techniques de } \\
\text { collecte des } \\
\text { données }\end{array}$ \\
\hline $\begin{array}{l}\text { L'analyse consistera à retracer la dynamique de l'évolution } \\
\text { des RTE; et identifier les types de réseaux: réseau incomplet } \\
\text { «lorsqu'une ou plusieurs catégories d'acteurs qui le } \\
\text { constituent... sont presque totalement absentes ou sous- } \\
\text { développées»; réseau chaîné «Si... tous les acteurs et les } \\
\text { pôles sont en position et fortement structurés»; établir le } \\
\text { degré d'intégration du réseau par l'analyse de sa } \\
\text { «dispersion», si sa densité est faible ou élevée, ou sa } \\
\text { «convergence», si elle est faible ou forte; qu'ils soient longs } \\
\text { ou courts en fonction de l'exhaustivité des RTE }\end{array}$ & $\begin{array}{l}\text { Quantitatives et } \\
\text { qualitatives } \\
\text { combinant les } \\
\text { outils } \\
\text { quantitatifs et } \\
\text { qualitatifs des } \\
\text { étapes 1-4. }\end{array}$ \\
\hline
\end{tabular}

\section{Conclusion}

Le present article propose un guide méthodologique pouvant être utilisé comme guide de diagnostic, des analyses et des études des SIN en Afrique. Il ressort de nos travaux et de la littérature qu'une contextualisation des outils fournis par le modèle est nécessaire. Pour relever ce défi méthodologique nous avons fait l'état des approches utilisées sur l'innovation agricole au Burkina Faso : l'approche intégrée autour des cultures de rente, l'approche formation et visite, l'approche plateforme d'innovation, l'approche système d'innovation. Ces différentes approches ont toutes été implémentées dans plusieurs pays africains mais surtout au Burkina Faso. Pour étudier un SIN, nous avons proposé et caractérisé six étapes essentielles de diagnostic adaptées au contexte des pays africains, surtout ceux partageant des contextes macro-économiques similaires au Burkina Faso. Cette proposition méthodologique de diagnostic des systèmes d'innovation agricoles en Afrique s'inscrit dans le cadre de travaux plus globaux pour documenter les experiences Africaines.

\section{References}

AKRICH, M., CALLON M. AND LATOUR B., 'The key to success in innovation Part I: the art of interessement'. International Journal of Innovation Management 6 (2): pp.187-206, 2002

BELLONCLE, G., 'Recherche, vulgarisation et Developpement rural en Afrique Noire'. Paris: Ministère de la Coopération, 1987

BJARAS, G., HAGLUNG A., J., B. AND KIFKIN B., S., 'A New Approach to Community Participation Assessment'. Health Promotion International 6(3): pp.199-206, 1991

CALLON, M., Techno-economic networks and irreversibility. In: Law, J. (Ed.), A Sociology of Monsters: Essays on Power, Technology and Domination. Routledge, London, pp. 132-161, 1991

CALLON, M. AND MUNIESA, F., 'Les marchés économiques comme dispositifs collectifs de calcul', Réseaux, 21(122),189-233, 2003 
CALLON M., LAREDO P., MUSTAR P., 'La gestion stratégique de la recherche et de la technologie', Paris : Economica, 1995

CALLON, M. LAREDO P. AND RABEHARISOA V., 'The management and evaluation of technological programs and the dynamics of techno-economic networks:The case of the AFME', Research Policy, 21, 215-236, 1992

CASADELLA, V, BENLAHCEN-TLEMCANI, M. 'De l'applicabilité du Système National d'Innovation dans les Pays Moins Avancés'. Innovations, (2), 2006, 59-90.

CIMOLI, M., DOSI, G., \& STIGLITZ, J. E., 'Industrial policy and development: The political economy of capabilities accumulation'. Oxford: University Press. 2009

CIRAD-GRET., 'Faciliter l'émergence et la diffusion des innovations' In : Memento de l'agronome, Ouagadougou : CIRAD pp. 373-405., 2002

COMPAORE E. M.F.W., 'science, technology and development: case study of agricultural biotechnology in Burkina Faso', Institute for Science and Society, University of Nottingham, United Kingdom (Registered as an MA by Research in Science, Technology and Society), 2009-2010

COMPAORE M.F.W E., 'The Role of the National Innovation Systems Framework Facilitating in Socio-Economic Development in Burkina Faso: Model and Policy Practice', PhD thesis, University of Nottingham, http://citeseerx.ist.psu.edu/viewdoc/download?doi=10.1.1.329.2666\&rep=rep1\&type=pdf, 2015

DAKOUO, D., 'Document national de synthese des etats des lieux des resultats de recherché et des innovations du Burkina Faso'. Ouagadougou: MESSRS, IDR., 2008

DUFUMIER M., Les Projets de Developpement Agricole:Manuel d'expertise, CTA-Karthala. 1996

EDQUIST, C., Systems of innovation approaches-their emergence and characteristics. In Edquist, C. (ed). Systems of Innovation Technologies, Institutions and Organisations. London: Pinter., 1997

EVANSON, E. R., Recherche, Vulgarisation et Productivité Agricole en Afrique de L'Ouest. In :

Belloncle, G. (Ed.) Recherche, vulgarisation et Développement rural en Afrique Noire, Paris : Ministère de la Coopération characteristics. In Edquist, C. (ed). Systems of Innovation Technologies, Institutions and Organisations. London: Pinter., 1987

FEINSON, S., National innovation systems overview and country cases. working paper. Center for Science, Policy, and Outcomes. Columbia University Washington, DC, and School of Public Policy, Georgia Tech, Atlanta, GA., 2002

FEINSON S., National Innovation Systems, Overview and Country Cases. Knowledge Flows, Innovation and Learning in Developing Countries 13 (38) Paris: OECD., 2003

FREEMAN, C., Technology infrastructure and international competiveness, draft paper submitted to the OECD ad hoc group on Science, Technology and Competiveness, august 1982.

FREEMAN, C. Technology Policy and Economic Performance: Lessons from Japan. London: Pinter., 1987

FREEMAN, C., LUNDVALL, B. Small Countries Facing the Technological Revolution. Pinter : London., 1988

FREEMAN, C.. "The 'National System of Innovation'in historical perspective." Cambridge Journal of economics19(1): 5-24., 1995

GAILLARD, J., La coopération scientifique et technique avec les pays du Sud: peut-on partager la science?, Paris : Karthala Editions., 1999

GLIN, C. L. Analyse des Cas : Platforms Multi-Acteurs, Réalité ou Nouvelle Trouvaille Sémantique? In : Vodouhê G. F., Lançon J., Vodouhê, S. D. (Eds.) (2010) Les plates-formes multi-acteurs dans le système national de la recherche agricole du Bénin, pp. 31-37. Actes de l'atelier, 19-20 octobre 2010. Cotonou. Bénin. OBEPAB: Coopération française., 2010

GODIN, B., "National innovation system the system approach in historical perspective." Science, Technology \& Human Values34(4): 476-501., 2009

GOTO, A., Japan's National Innovation System: Current Status and Problems. Oxford Review of Economic Policy16 (2): pp.103-113., 2000 
KATZ E. RIAZ, J. DE MEYER, B. DOSOV AND K. NICHTERLEIN, Approaches to Strengthening Agricultural Innovation Systems (Ais) In Central Asia, South Caucasus And Turkey: Insights Based on Assessments of Strengths and Weaknesses of the AIS in Kyrgyzstan, Tajikistan, Uzbekistan, Azerbaijan and Turkey, FAO, 2014

KOUTSOURIS A., Facilitating Agricultural Innovation Systems: a critical realist approach, Studies in Agricultural Economics 114 64-70, 2012

LUNDVALL, B.-A., Product Innovation and User-producer inter-action. Aalborg: Aalborg University Press, 1985

LUNDVALL, B.-A., National systems of Innovation: Towards a Theory of Innovation and Interactive learning. London: Pinter., 1992

LUNDVALL, B.-A., National Innovation Systems-Analytical Concept and Development Tool. Industry and Innovation 14 (1): pp.95-119. 2007

LUNDVALL B. A. and BORRAS, Implication for Innovation Policy. Science and Public Policy 31 (6). 1997

MUCHIE, M. GAMMELTOFT, P. and LUNDVALL, A., B., Putting Africa First, the Making of Africa Innovation Systems. Aalborg : Aalborg University Press, 2003

MANLEY, K., "Frameworks for understanding interactive innovation processes." The International Journal of Entrepreneurship and Innovation4(1): 25-36., 2003

MORRISON, M. AND MORGAN, S. M., Models as Mediators, perspective in natural and social science. Cambridge: Cambridge University Press, 1999

MIETTENEN, R., Innovation Human Capabilities and Democracy. Oxford: University Press, 2013

MESSRS, Symposium National de Farako-Ba : Une Recherche Scientifique de Masse au Service du Développement Independent, autosuffisant et Planifie du Burkina Faso. Rapport de Synthèse, Ouagadougou : CNRST, 1987

MRSI, Politique Nationale pour la Recherche Scientifique et Technique (PNRST) 2013-2025. Ouagadougou: MRSI, 2012

MESSRS, Forum National Sur la Recherche Scientifique au Burkina Faso, Document de Base : Situation Institutionnelle de la Recherche Scientifique et Technologique au Burkina Faso. Ouagadougou : MESSRS, 1994

MESSRS, Memorandum sur la situation à l’Université de Ouagadougou. Ouagadougou : UO., 1999

MESSRS, rapport de l'état des lieux auprès des départements ministériels, des 13 regions et des partenaires stratégiques et limitrophes du Burkina Faso dans le cadre du projet. Ouagadougou : FRSIT, 2008

MILLOGO, C.. Plateforme d'innovation multi-acteurs pour le transfert de technologique comme facteur d'amélioration de 1 performance agronomique chez le producteurs au Burkina Faso : cas des entrepreneurs agricoles de la Sissili, mémoire de fin cycle, Mémoire de Maitrise, Universite de Ouagadougou, 2013

MORRISON, M. AND MORGAN, S. M., Models as Mediators, perspective in natural and social science. Cambridge: Cambridge University Press, 1999

NATIONS UNIES, Investment Policy Review of Burkina Faso. New York : Nations Unies, 2009

NAULEAU, G., Une Operation de Motorisation Intermediaire au Burkina Faso. In : Belloncle G. (Eds.) Recherche, vulgarisation et Developpement rural en Afrique Noire, 1987. Paris: Ministere de la Cooperation, 1987

NELSON, R. R., National innovation systems: a comparative analysis. Oxford: University Press, 1993

OECD, Managing National Innovation Systems. Paris: OECD Publications, 1999

OCDE, Les systèmes d'innovation agricole. Cadre pour l'analyse du rôle des pouvoirs publics. Paris : OCDE, 2013

PICKERING, C. D., l'Expérience de la Banque Mondiale en Matière de Vulgarisation Agricole. In : Belloncle G. (Ed.) Recherche, Vulgarisation et Developpement Rural en Afrique Noire, 1987. Paris: Ministere de la Cooperation, 1987

RUSSELL, J., La Banque Mondiale et la Vulgarisation Agricole : L'Introduction du Systems Formation and Visites en Afrique Subsaharienne. In : Belloncle, G. (Ed.) Research, Vulgarisation et Developpement Rural en Afrique Noire, 1987. Paris : Ministere de la Cooperation, 1987

SAWADOGO N. AND SAWADOGO/COMPAORE E. M.F.W, Theorising Africa's Development Problem, in Olanyinka A. and Adesina J. O., The Development of Africa: Issues, Diagnoses and Prognoses, Springer International Publishing; p55-6, 2018 
SAWADOGO/COMPAORE E. M.F.W., Transition Politique et Accumulation Capitaliste : Le Cas du Commerce du Coton au Burkina Faso Post Révolutionnaire, in Sanon F et Sissao A. J., Développement Endogène de l'Afrique et Mondialisation: Une Relecture de la Pensée du Professeur Joseph Ki-Zerb, PUO : Fondation Joseph Ki-Zerbo, p705-728. 2016

SAWADOGO/COMPAORE E. M.F.W., Monsters in agriculture: Moral controversy around Genetically Modified cotton in Burkina Faso, in Kouadio Germain N'GUESSAN (ed) Writing abnormality/Écriture de l'anormalité, a collective book, Pressse Universitaire de Lome, Chap 4, p 40-50. 2016

SAWADOGO/COMPAORE E. M.F.W, More Pieces Within the Peaces: Rethinking the Financial Systems for Agricultural Innovation in Burkina Faso, Science and Technology Policy Research Institute Innovation Conference (ICGhana 2016) Theme: "Development Innovation - Putting the Pieces Together". Date: 27th-28th Sept. 2016; Venue: La Palm Royal Beach Hotel, Accra, 2016

SAWADOGO/COMPAORE E. M.F.W., Le Cotton Biotechnologique au Burkina Faso : Trajectoire d'une Innovation dans un Contexte de Développement, numéro spécial de technologie innovation sur l'innovation agro-écologique et de développement, 'Innovation Agro-Ecologiques et Developpement' (2) 27-37, London, UK - openscience.fr, 2017

SAWADOGO/COMPAORE E. M.F.W AND SAWADOGO N., Capacity Development for Agricultural Innovation Systems in Burkina Faso: What's New with CDAIS Project? In Kebe C.M.F., Gueye A., Ndiaye A. Innovation and Interdisciplinary Solutions for Underserved Areas, EAI: Springer International Publishing, 2018

SHARIF, N., Emergence and development of the National Innovation Systems concept. Research Policy 35 (5): pp. 745-766. 2006

SHARIF, N., Rhetoric of Innovation Policy Making in Hong Kong Using the Innovation Systems Conceptual Approach. Science, Technology and Human Value. http://sth.sagepub.com/content/early/2009/05/29/0162243909337120, 2009

SHARIF, N., Contributions from the Sociology of Technology to the Study of Innovation Systems. Knowledge, Technology, \& Policy 17 (3-4): pp. 83-105, 2004

TOUZARD J-M, TEMPLE L., FAURE G., TRIOMPHE B., Innovation Systems and Knowledge Communities in the Agriculture And Agrifood Sector: A Literature Review, Journal of Innovation Economics \& Management, $\mathrm{n}^{\circ} 17$, $117-142,2015$

UNESCO, Science for the Twenty-first Century: A New Commitment, World Conference on Science,1999, Paris, UNESCO, 2000

WORLD BANK, Knowledge for Development. Washington DC: World Bank, 1999 